\title{
Design and Fabrication of 3-DOF Robot Arm Using Parallelogram Mechanisms
}

\author{
Vo Duy Cong ${ }^{1,2}$, Dang Anh Duy ${ }^{1,2}$, Le Hoai Phuong ${ }^{1,2}$ \\ ${ }^{1}$ Ho Chi Minh City University of Technology (HCMUT), 268 Ly Thuong Kiet, District 10, \\ Ho Chi Minh City, Viet Nam \\ congvd@hcmut.edu.vn, aduy@hcmut.edu.vn,lhphuong@hcmut.edu.vn \\ ${ }^{2}$ Vietnam National University Ho Chi Minh City (VNU-HCM), Linh Trung Ward, Thu Duc District, \\ Ho Chi Minh City, Vietnam
}

Received Date : August 03, 2021 Accepted Date : August 22, 2021 Published Date : September 07, 2021

\begin{abstract}
This paper focuses on the design, fabrication and control of a 3 -DOF robot arm using stepper motors. The robot arm uses three parallelogram mechanisms to position the end-effector of the robot and keep the end-effector always parallel to the horizontal during the robot motion. The robot is designed on the Autodesk Inventor software. Separated parts of the robot are saved in the stereolithography (STL) file format. Then the parts are fabricated by a 3D printer. The movement of the robotic arm is driven by stepper motors and controlled by Arduino. The Arduino board implements kinematics calculation, creates pulses and sends them to three drivers to driven stepper motors. A software is developed to control the robot by sending the command to the Arduino board.
\end{abstract}

Key words: Robot arm, parallelogram mechanisms, robot controller, 3D printing.

\section{INTRODUCTION}

In recent decades, automation in production has become more and more popular, not only in large factories but also in small and medium factories, Automatic machines are gradually replacing manual steps in the production process to increase productivity and product quality. A robot is one of the automatic devices to replace humans in the production process.

Because of the ability to perform dangerous, dirty and/or repetitive tasks with consistent precision and accuracy, the industrial robot arm is increasingly used in a variety of industries and applications such as handling, palletizing, cutting, finishing, sealing and gluing, spraying, welding [1-4].

In this paper, we present a design and fabrication of a 3-DOF robot arm using parallelogram mechanisms to position the end-effector. This type of robot is widely used in many industrial applications such as picking, packing, sorting, palletizing, ... The mechanical model of the robot is designed on the Autodesk Inventor software. The separated parts of the robot are fabricated on an Anycubic i3 Mega 3D printer. The movement of the robotic arm is driven by gearbox stepper motors. Arduino Uno board is selected for robot control. Arduino receives commands from the control app and outputs control signals to stepper motors.

\section{LITERATURE REVIEW}

Kruthika et al. [5] present the design and development of a 5-DOF robotic arm used for feeding the elderly or specially challenged people. The joints angle of the robot is controlled by the user by using principles of robotic Kinematics and MATLAB. Arduino MEGA2560 board is the main heart of this project which interfaces with the Graphical User Interface, motors and sensors. The accuracy of the kinematics algorithm is verified by MATLAB simulation.

In [6], a robotic arm for rehabilitation and training is developed. This wearable robot arm can be used easily with both arms of users. Each joint has a range of rotation from -90 to 90 degrees so can cover all motions of the human arm. The designed robot arm will support the patient's arm during training which is repetitive tasks and takes some period of time.

Kumra et al. [7] present the design and development of a low-cost and user-friendly interface for the control of a 6-DOF slave teleoperated anthropomorphic robotic arm. The robot arm is driven by six servo motors. A 2 DOF gripper is designed for grasping objects. Force sensors are mounted between the joints of the manipulator to measure the amount of strain placed on each of these joints. Various experiments are performed to check the performance and the accuracy Man-Machine Interface. The robot can achieve position precision within $\pm 0.5 \mathrm{~cm}$. 
Vo Duy Cong et al., International Journal of Emerging Trends in Engineering Research, 9(9), September 2021, 1224 - 1229

Sreekanth et al. [8] develop a 6 DOF manipulator used in an alien environment and for space exploration. The robot is made with advanced composite materials with feature lightweight, high precision. The arm has 2 types of end effectors in an adaptive manner so that it can be utilized in grasping both symmetric to asymmetric objects. The high configurability of this arm enables it to perform different tasks efficiently under a single platform in space applications.

A design and development of 5-DOF robot arm manipulators are presented in [9]. the robotic arm's angles are controlled by using Cortex ARM M3 LPC1768 Microcontroller. The servo motor is situated at each joint to position the link that is required at the particular angle using position-controlling on the controller. By using rotary encoders, the feedback of the angle can be measured. The major advantage of the robotic arm is that it can work in high temperature and pressure environments which is not suitable for humans. The operation of the designed robotic arm is verified by experiment.

In [10], Huang et al designs and controls an automated arm, which is mounted on the portable robotic body. The robotic arm is controlled by the $8052 \mathrm{MCU}$ of Atmel arrangement. The authors have presented the experimental results that the developed system of dual robotic arms can get organizes from binocular vision and hold the payload. This investigation finished a sign transmission, the speed and rakish control from PC to double automated arm framework.

\section{ROBOT KINEMATICS}

Figure 1 shows the mechanical schematic of the robot arm. The end-effector of the robot is positioned using three parallelogram mechanisms that are composed of the following sets of links: $\{1,2,4,6\},\{0,1,3,5\}$ and $\{5,6,7,8\}$. For every motion of the robotic arm the links 0,5 and 8 remain parallel to each other and thus keep the end-effector (link 8) always parallel to the horizontal.

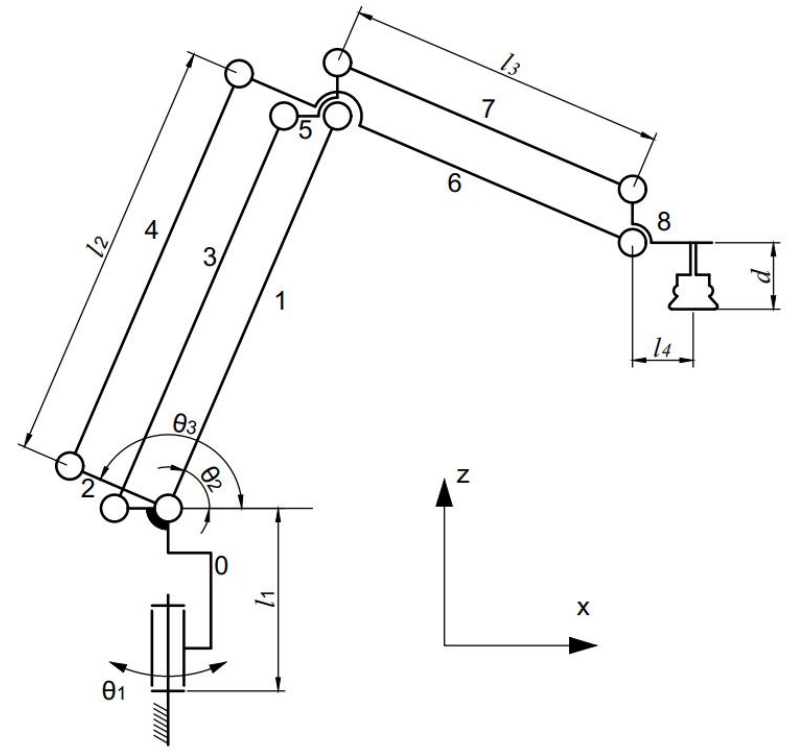

Figure 1: The robot arm schematics
The robot has three degrees of freedom corresponding to the rotation angles $\theta_{1}, \theta_{2}$ and $\theta_{3}$. Three stepper motors that placed at each joint control movements of links 0,1 and 2 and these motions are planned to create the desired motion of the end-effect or. Before planning the trajectory, it is necessary to determine the value of the joint angles by solving the kinematics problem.

From basic trigonometry, the position of the end effect or can be written in terms of the joint angles as follows:

$$
\begin{gathered}
x=\cos \theta_{1}\left(l_{2} \cos \theta_{2}-l_{3} \cos \theta_{3}+l_{4}\right) \\
y=\sin \theta_{1}\left(l_{2} \cos \theta_{2}-l_{3} \cos \theta_{3}+l_{4}\right) \\
z=l_{2} \sin \theta_{2}-l_{3} \sin \theta_{3}+l_{1}-d
\end{gathered}
$$

Equations (1), (2) and (3) are called forward kinematic equations of the robot manipulator that describe the relationship between the end-effect or coordinates and joint angles. To find the joint angles for a given set of end-effect or coordinates, one needs to solve the inverse kinematic equations.

From (1) and (2), we easily obtain:

$$
\theta_{1}=\operatorname{atan} 2(y, x)
$$

Here we used the atan 2 function to get the unique joint angle $\theta_{1}$. Square both sides in (1) and (2) then add them together:

$$
l_{2} \cos \theta_{2}-l_{3} \cos \theta_{3}+l_{4}= \pm \sqrt{x^{2}+y^{2}}
$$

Combine with(3) and group the unknowns on the left-hand side:

$$
\begin{gathered}
l_{2} \cos \theta_{2}-l_{3} \cos \theta_{3}= \pm \sqrt{x^{2}+y^{2}}-l_{4}=a \\
l_{2} \sin \theta_{2}-l_{3} \sin \theta_{3}=z+d-l_{1}=b
\end{gathered}
$$

Square both sides in each equation and add them together. After that rearrange theterms, we get an equation in $\theta_{3}-\theta_{2}$ :

$$
l_{2}^{2}+l_{3}^{2}-2 l_{2} l_{3} \cos \left(\theta_{3}-\theta_{2}\right)=a^{2}+b^{2}
$$

Now, we can obtain the angle $\theta=\theta_{3}-\theta_{2}$ :

$$
\theta=\theta_{3}-\theta_{2}= \pm \operatorname{arcos} \frac{l_{2}^{2}+l_{3}^{2}-a^{2}-b^{2}}{2 l_{2} l_{3}}
$$

Rearranging (7) according to the unknown angle $\theta_{2}$, we get:

$\left(l_{2}+l_{3} \cos \theta\right) \sin \theta_{2}-l_{3} \sin \theta \cos \theta_{2}=z+d-l_{1}=b$

Definer and $\varphi$ so that:

$$
\begin{gathered}
r=\sqrt{\left(l_{2}+l_{3} \cos \theta\right)^{2}+\left(l_{3} \sin \theta\right)^{2}} \\
\sin \varphi=\frac{l_{2}+l_{3} \cos \theta}{r} \\
\cos \varphi=\frac{l_{3} \sin \theta}{r}
\end{gathered}
$$

The angle $\varphi$ can be determined by using the atan 2 function: 
Vo Duy Cong et al., International Journal of Emerging Trends in Engineering Research, 9(9), September 2021, 1224 - 1229

$$
\varphi=\operatorname{atan} 2\left(\frac{l_{2}+l_{3} \cos \theta}{r}, \frac{l_{3} \sin \theta}{r}\right)
$$

Substituting $r$ and $\varphi$ into (10) we get:

$$
\cos \left(\theta_{2}+\varphi\right)=-\frac{b}{r}
$$

Finally, the solution of angle $\theta_{2}$ is:

$$
\theta_{2}= \pm \operatorname{arcos}\left(-\frac{b}{r}\right)-\operatorname{atan} 2\left(\frac{l_{2}+l_{3} \cos \theta}{r}, \frac{l_{3} \sin \theta}{r}\right)
$$

There are four solutions for a given end-effector position. That means there are four configurations that the robot must choose to reach the desired position. The value of $\theta_{1}$ is unique, the practical joint limits of joints 2 and 3 are used to get the unique configuration:

$$
\begin{gathered}
-\frac{\pi}{2} \leq \theta_{2} \leq \frac{\pi}{2} \\
0 \leq \theta_{3}-\theta_{2} \leq \pi
\end{gathered}
$$

\section{MECHANICAL DESIGN AND FABRICATION}

\subsection{Robot base design}
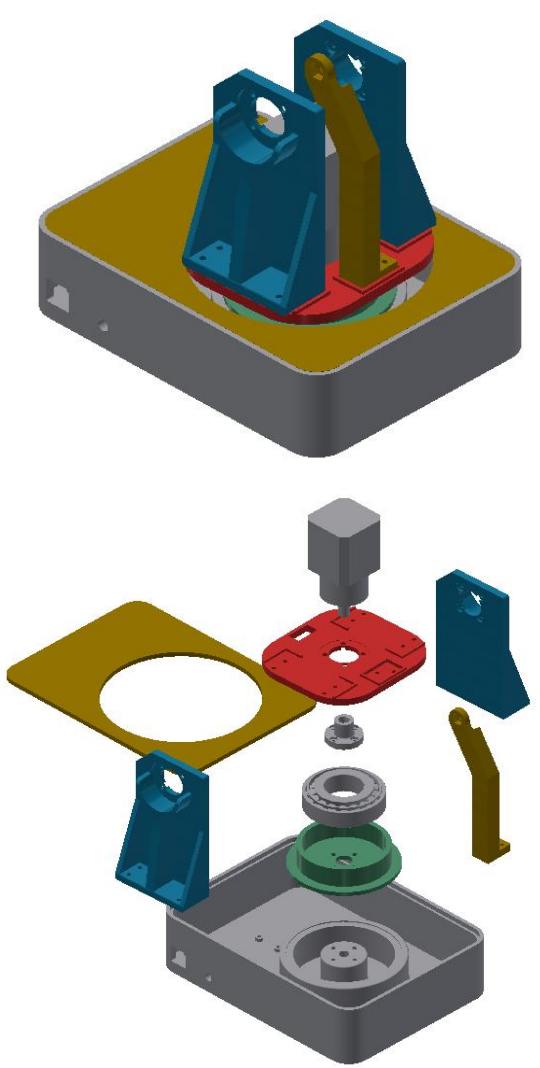

Figure 2: The robot base design

The base is an important component that supports the total weight of the robot. Figure 1 shows parts of the robot base. A tapered roller bearing is placed between the motor of joint 1 and the base, the bearing bears all the weight of the robot, creating a solid structure. Two parts with support ribs on both sides are attached to stepper motors, linking the base to the upper arm.

\subsection{Parallelogram mechanisms design}

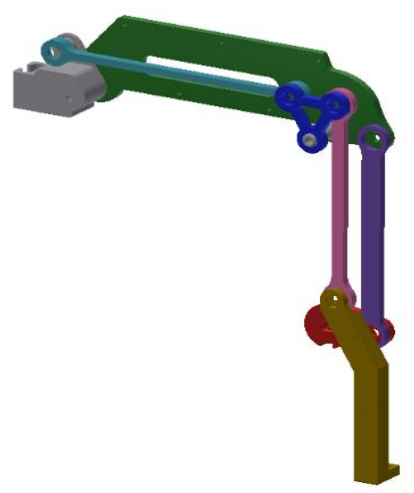

Figure 3: The robot base design

From the schematics in Figure 1, the parallelogram mechanisms are designed as shown in Figure 3. The links in mechanisms are represented by flat bars with a thickness of $5 \mathrm{~mm}$. The two ends of each bar are fitted with ball bearings so that they can be linked to other links and create relative motion. The length of links is as follows: $l_{2}=135 \mathrm{~mm}, l_{3}=$ $150 \mathrm{~mm}$

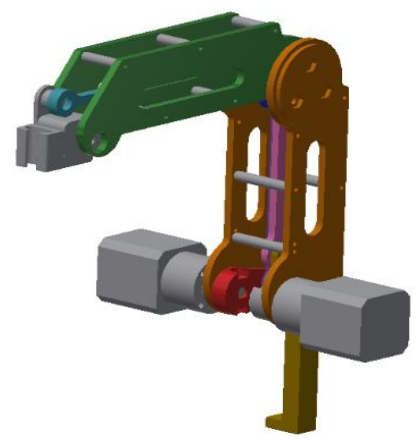

Figure 4: The upper arms design

The arms are designed as flat plates and connected by cylindrical steel rods.Figure 4 shows the upper arms when assembling the flat plates and the stepper motors.

Finally, the base and the upper arm are assembled together to form the complete model as shown in Figure 5.

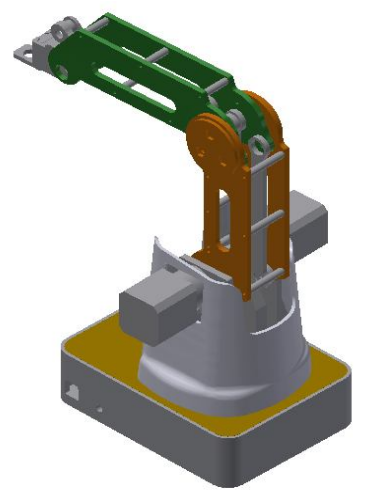

Figure 5: The complete model design 


\subsection{D printing}

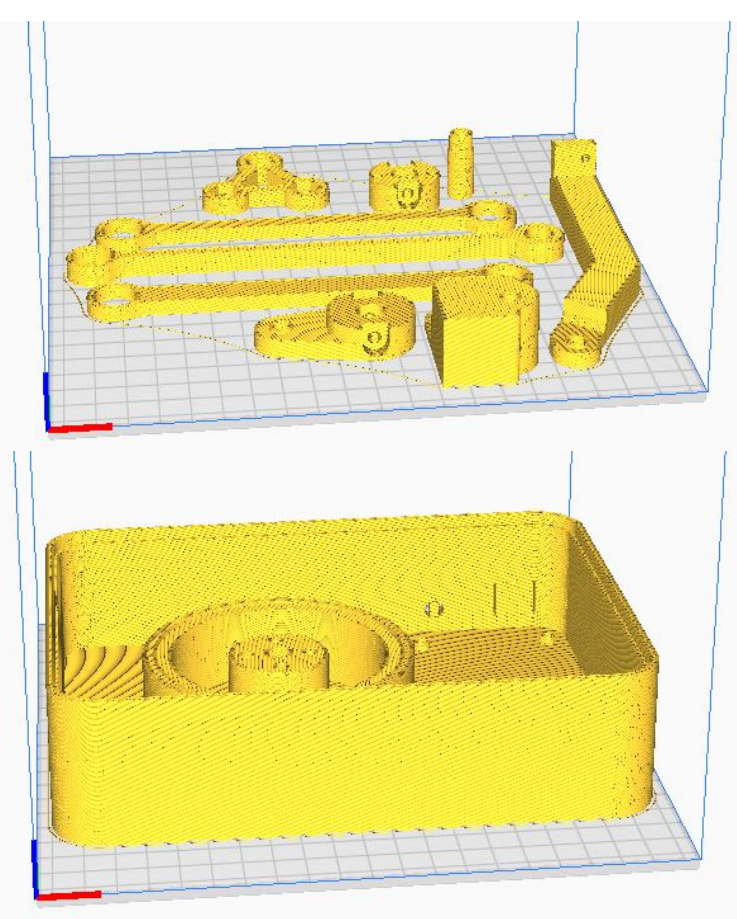

Figure 6: Slicing on Cura Software

Separated parts of the robot are exported in the stereolithography (STL) file format. The STL files are imported to the Cura slicing software where they are sliced and output as G-Code, which is the code that a 3D printer understands. Figure 6 shows some slice models on the Cura software. Then the parts are fabricated by a 3D printer. Any cubic i3 Mega printer machine is used.

Finally, the 3D printed components are assembled together and with other parts (e.g., motors, bearings, shafts, ...) to produce a complete robot arm as shown in Figure 7.

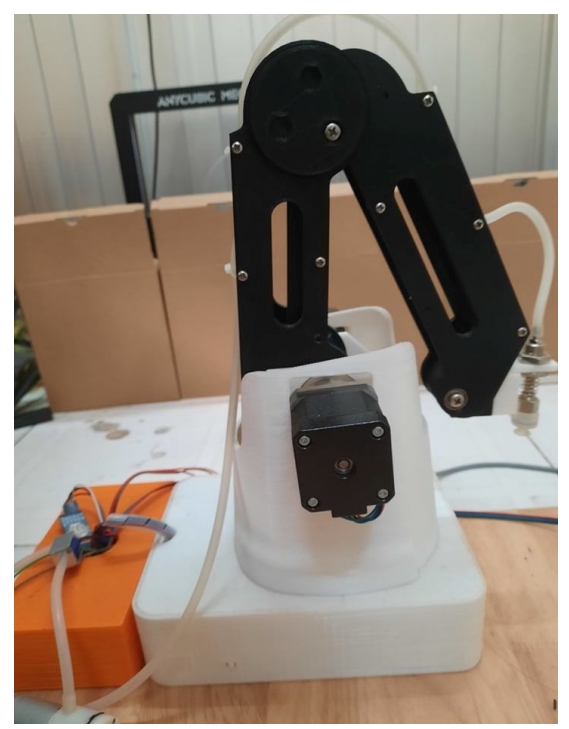

Figure 7: Actual robot arm

\section{ELECTRONIC AND SOFWARE DESIGN}

\subsection{Electronic design}

Figure 8 shows the block diagram of the electronic circuit. The Arduino board creates pulses and sends them to three A4998 drivers to driven stepper motors. In addition, the Arduino also outputs digital signals to control the solenoid valve, pump and motor. Since the valve and pump operate at $12 \mathrm{~V}$, relays whose coils are energized by the $5 \mathrm{~V}$ signal from the Arduino are used to turn ON and OFF them. The pump, solenoid valve and vacuum suction cup are used in a vacuum system to grip objects. Arduino communicates with the app on mobile phone via Bluetooth using the module HC-06 (slave). Bluetooth communication can be used to control the robot remotely.

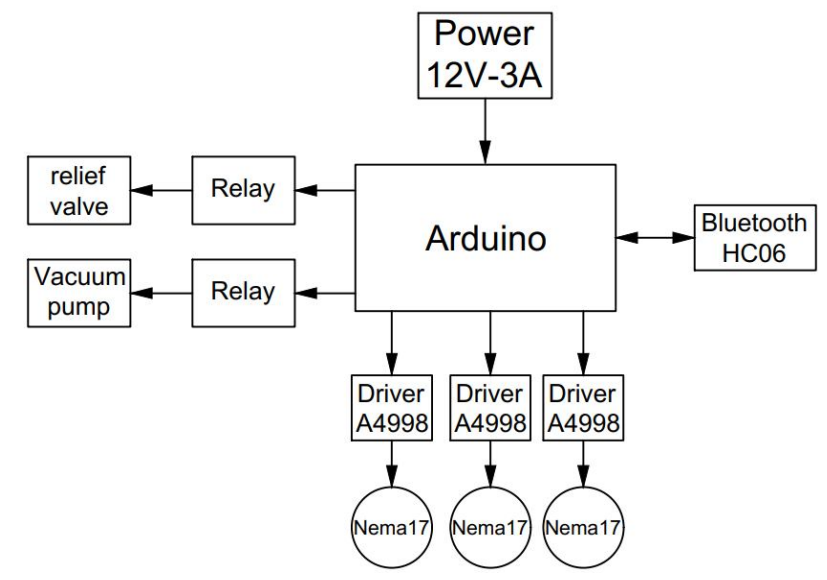

Figure 8: The block diagram of electronic circuit

The joints of the robot arm are driven by 5.18:1 planetary gearbox stepper motors (Figure 9). The motor has a step angle of 1.8 degrees, a rated current of $1.3 \mathrm{amp}$, holding torque of 28 N.cm. The A4998 drivers are set up to operate at the $1 / 16$ step resolution.

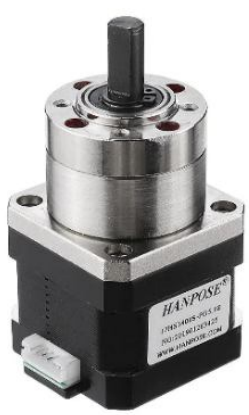

Figure 9: 5.18:1 planetary gearbox stepper motors

For simplicity of wiring, we use a CNC shield to connect Arduino and stepper drivers as shown in Figure 10. The actual circuit is placed on a box with dimensions of $150 \times 100 \times 50 \mathrm{~mm}$ as shown in Figure 11. 


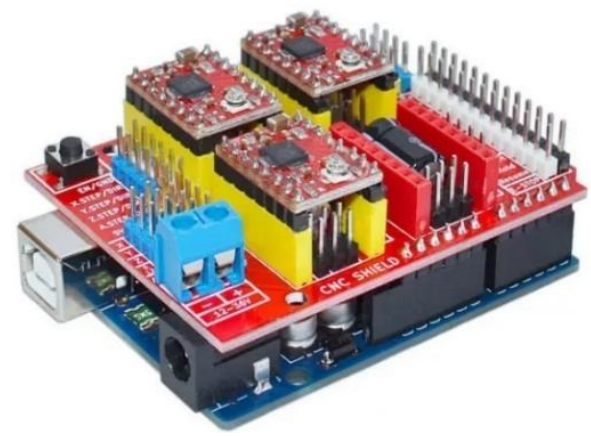

Figure 10: CNC shield

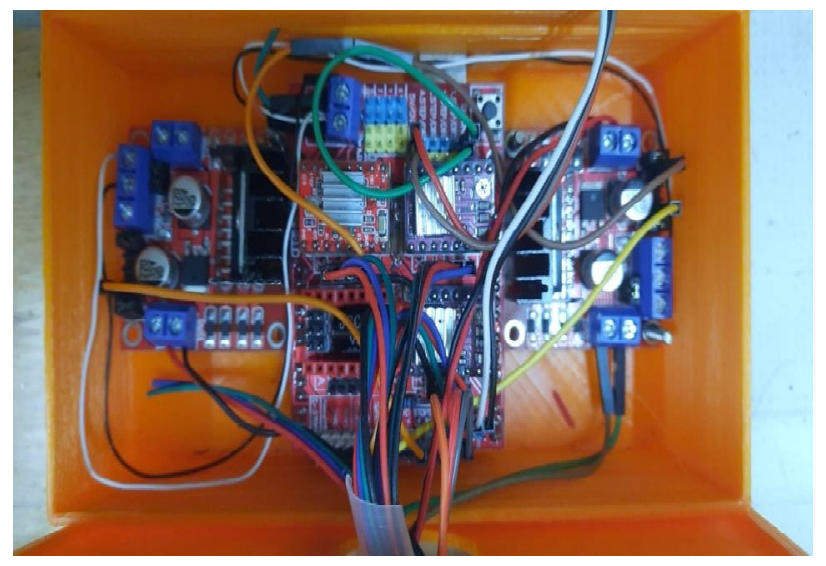

\subsection{Mobile app design}

Figure 11: Actual circuit

The mobile app is designed on the MIT Inventor app. The interface screen is shown in Figure 12. The "+" buttons are used to increase the angle value by 0.5 degrees, The "-" buttons are used to decrease the angle value by 0.5 degrees. Drag the sliders to change the joint angle quickly. Every time there is a change of joint angles, the new value is sent to the Arduino via Bluetooth.

\section{Đại học Bách khoa Tp.HCM TT Đào tạo Bảo dưỡng Công nghiệp \\ Robot Arm}

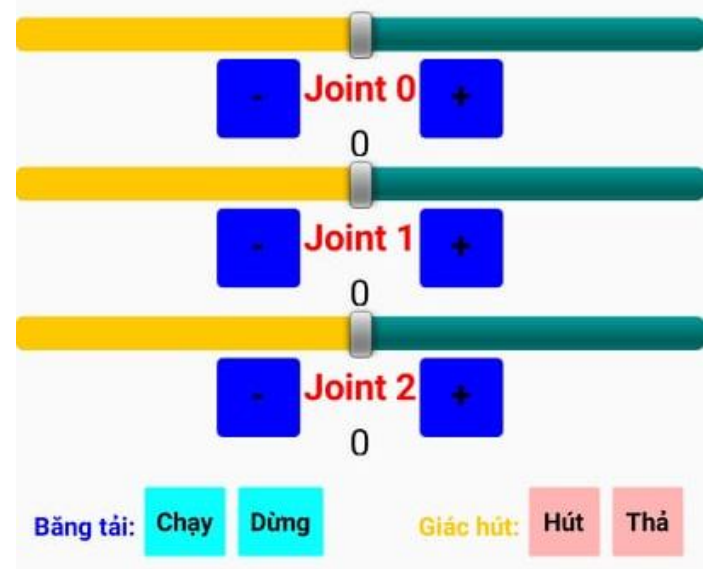

Figure 12: Mobile app screen

The app is programmed by using the block-structured programming language. We program the app by putting blocks together. Each block has a behavior corresponding to a specific function. The blocks are combined in a logical sequence to perform the desired function. Figure 13 shows the block code of the app to control the robot arm.
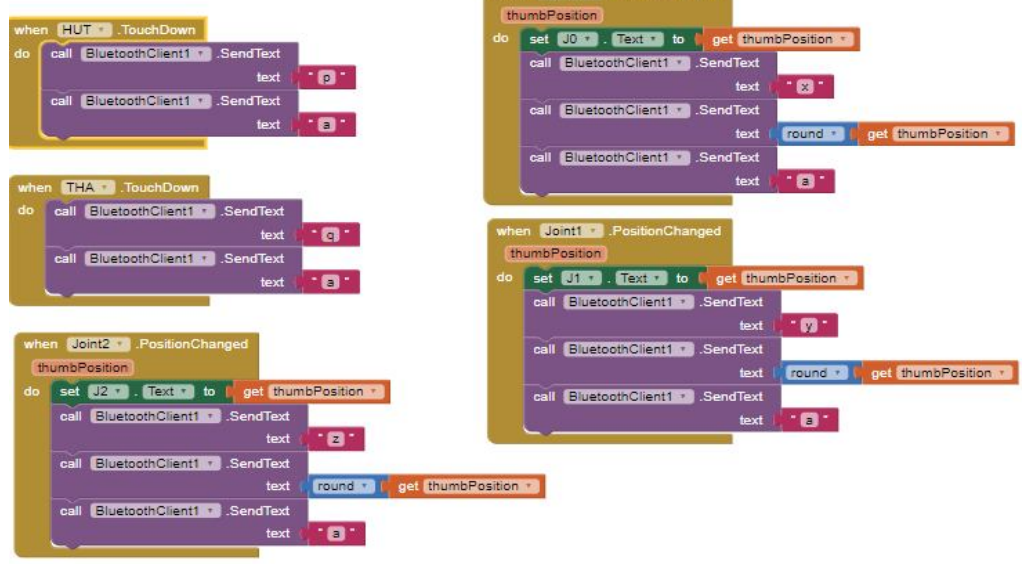

Figure 13: Block design on MIT Inventor

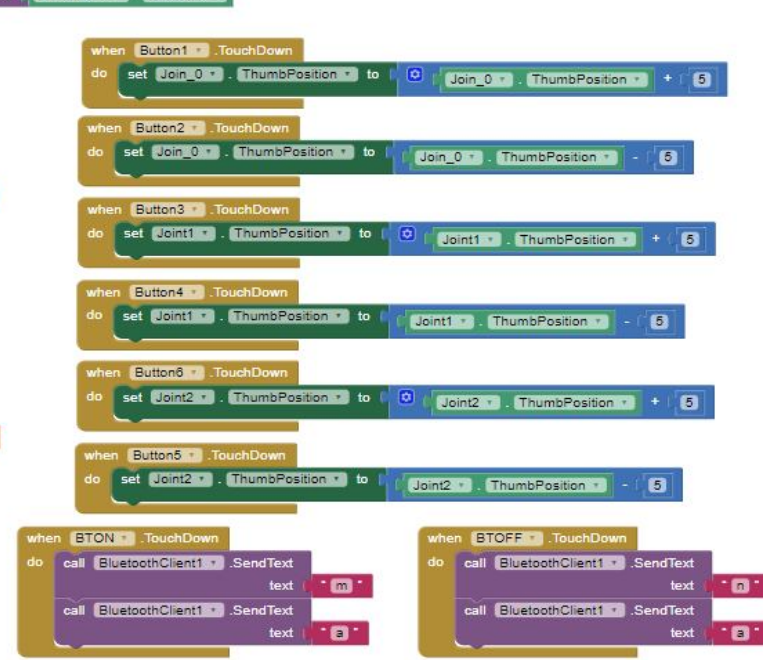


Vo Duy Cong et al., International Journal of Emerging Trends in Engineering Research, 9(9), September 2021, 1224 - 1229

\section{CONCLUSION}

In this paper, a 3-DOF robot arm is designed and fabricated. The mechanical models are designed on Inventor software and export to the STL file for 3D printing. Using Arduino board and CNC shield has made the circuit design and wiring process simpler. The mobile app is developed to control the robot arm through Bluetooth.

\section{ACKNOWLEDGEMENT}

We acknowledge the support of time and facilities from Ho Chi Minh City University of Technology (HCMUT), VNU-HCM for this study.

\section{REFERENCES}

1. H. F. Fauadi and M. S. Jumali."Modelling and simulation of programmable universal machine for assembly (PUMA) industrial robot for automotive related assembly process", International Symposium on Information Technology, pp. 1-5, 2008

2. G. S. Huang, C. K. Tung, H. C. Lin, and S. H. Hsiao, "Inverse kinematics analysis trajectory planning for a robot arm", Control Conference, pp. 965-970, 2011.

3. E. S. Kheng, A. H. A. Hassan, A. Ranjbaran, and T. S. Siong,"Range estimation for robot arm applications using image segmentation and curve fitting tool",International Conference on Electrical, Control and Computer Engineering, pp. 275-278, 2011.

4. X. Chu, H. Fleischer, N. Stoll, M. Klos, and K. Thurow. "Application of dual-arm robot in biomedical analysis: Sample preparation and transport",Instrumentation and Measurement Technology Conference, pp. 500-504, 2015.

5. K. Kruthika,B. M. Kiran Kumar and S. Lakshminarayanan, "Design and development of a robotic arm",2016 International Conference on Circuits, Controls, Communications and Computing (I4C), pp. 1-4, 2016.

6. P.Sarut and C. Siam, "Design and Development of a Robotic Arm for Rehabilitation and Training",Lecture Notes in Electrical Engineering,pp. 3-8, 2017.

7. S. Kumra, R. Saxena and S. Mehta, "Design and development of 6-DOF robotic arm controlled by Man Machine Interface”, 2012 IEEE International Conference on Computational Intelligence and Computing Research, pp. 1-5, 2012.

8. N. Sreekanth, A. Dinesan, A. Nair, G. Udupa and V. Tirumaladass, "Design of robotic manipulator for space applications",Materials Today: Proceedings, Vol. 46, 2020

9. Y. Jadeja and B. Pandya, "Design and Development of 5-DOF Robotic Arm Manipulators", International Journal of Scientific \& Technology Research, Vol. 8, No.11, pp.2158-2167, 2019.
10. G.S Huang, X.S Chen and C.L Chang, "Development of dual robotic arm system based on binocular vision", Automatic Control Conference (CACS), CACS International, pp.97- 102,2013. 